INPLASY

PROTOCOL

To cite: Li et al. Efficacy and safety of adalimumab in the treatment of non-infectious uveitis: a meta-analysis of randomized controlled trials. Inplasy protocol 2020100116. doi:

10.37766/inplasy2020.10.0116

Received: 29 October 2020

Published: 30 October 2020

Corresponding author:

Biao Li

2037276404@qq.com

Author Affiliation:

Teaching hospital of Chengdu

University of Traditional

Chinese Medicine

Support: Province Department of science.

Review Stage at time of this submission: Preliminary searches.

Conflicts of interest:

None.

\section{Efficacy and safety of adalimumab in the treatment of non-infectious uveitis: a meta-analysis of randomized controlled trials}

\author{
Li, B1; Li, H²; Zhang, L3; Zheng, Y4.
}

Review question / Objective: P: patients have non-infectious autoim-mune uveitis; I: adalimumab; $\mathrm{C}$ : Conventional routine treatment; 0: Control of inflammation; Best-corrected visual acuity (BCVA); S: Randomized controlled trials (RCTs).

Condition being studied: Non-infectious uveitis describes a heterogenous group of ocular disorders of the anterior, intermediate, and posterior uveal tract (iris, ciliary body, choroid), characterised by intraocular inflammation in the absence of infection. Uveitis is the fifth most common cause of vision loss in high-income countries, accounting for $5 \%$ to $20 \%$ of legal blindness, with the highest incidence of disease in the working-age population. Treatment is aimed at reducing disease activity by suppression of the intraocular inflammatory response. Management includes local and systemic corticosteroids, immunosuppressants and biological drugs.

INPLASY registration number: This protocol was registered with the International Platform of Registered Systematic Review and Meta-Analysis Protocols (INPLASY) on 30 October 2020 and was last updated on 30 October 2020 (registration number INPLASY2020100116).

\section{INTRODUCTION}

Review question / Objective: P: patients have non-infectious autoim-mune uveitis; $\mathrm{I}$ : adalimumab; $\mathrm{C}$ : Conventional routine treatment; 0 : Control of inflammation; Best-corrected visual acuity (BCVA); S: Randomized controlled trials (RCTs).
Rationale: To summarize updated evidences on the efficacy and safety of adalimumab (ADA) in the treatment of patients with non-infectious uveitis.

Condition being studied: Non-infectious uveitis describes a heterogenous group of ocular disorders of the anterior, intermediate, and posterior uveal tract (iris, 
ciliary body, choroid), characterised by intraocular inflammation in the absence of infection. Uveitis is the fifth most common cause of vision loss in high-income countries, accounting for $5 \%$ to $20 \%$ of legal blindness, with the highest incidence of disease in the working-age population. Treatment is aimed at reducing disease activity by suppression of the intraocular inflammatory response. Management includes local and systemic corticosteroids, immunosuppressants and biological drugs.

\section{METHODS}

Search strategy: A relevant literature search will be conducted using the electronic databases of PubMed, Web of Seience, EMBASE, Cochrane, Library, clinicaltrials, CBM, CNKI, Wanfang and VIP from their inception to November 19, 2020, with no language restriction. We will use the following search terms: Uveitis, iridocyclitis, retinitis, retinal, vasculitis, panuveitis, Uveitides, uveit*; crossed with adalimumab, ADA, Humira, Amjevita, Cyltezo, D2E7, TNF, anti-tumornecrosis factor alpha, TNF-a inhibitors, anti-TNF-a. The search was restricted in the abstract। keywords title fields to cut down on irrelevant literature. Uveitis was not restricted by the words of "chronic", "noninfectious", and "autoimmune" to enlarge the number of hits in the literature to be screened.

Participant or population: § Participants of any age, gender or ethnicity with a diagnosis of non-infectious autoim-mune uveitis; § patients who were on a chronic course under immunosuppressive therapy and systemic steroid treatment previously for at least 3 months; $\S$ the uveitis inflammatory activity grading was described according to the Standardization of UveitisNomenclature (SUN) workinggroup criteria; § at least a 3-month median follow-up duration; § studies with at least five patients to avoid a positive report bias.

Intervention: Received adalimumab for the treatment of non-infectious uveitis.
Comparator: Conventional routine treatment(such as local and systemic corticosteroids, immunosuppressants).

Study designs to be included: Randomised controlled trials (RCTs).

Eligibility criteria: Study design § Randomised controlled trials (RCTs). Participants $\S$ Participants of any age, gender or ethnicity with a diagnosis of Non-infectious uveitis. Studies on a population broader than Non-infectious uveitis will only be included if data specific for the Non-infectious uveitis subgroup is reported separately. The uveitis inflammatory activity grading was described according to the Standardization of Uveitis Nomenclature (SUN) workinggroup criteria; at least a 3-month median follow-up duration; studies with at least five patients to avoid a positive report bias. patients received adalimumab for the treatment of non-infectious uveitis.

Information sources: A relevant literature search will be conducted using the electronic databases of PubMed, Web of Seience, EMBASE, Cochrane, Library, clinicaltrails, CBM, CNKI, Wanfang and VIP from their inception to September 2017, with no language restriction. We will use the following search terms: Uveitis, iridocyclitis, retinitis, retinal vasculitis, panuveitis, Uveitides, uveit ${ }^{\star}$; crossed with adalimumab, ADA, Humira, Amjevita, Cyltezo, D2E7, TNF, anti-tumornecrosis factor alpha, TNF-a inhibitors, anti-TNF-a. The search was restricted in the abstractlkeywords title fields to cut down on irrelevant literature. Uveitis was not restricted by the words of "chronic", "noninfectious", and "autoimmune" to enlargethe number of hits in the literature to be screened. Study types were limited to Randomised controlled trials (RCTs).

Main outcome(s): Intraocular inflammation remission.

Additional outcome(s): 1.Best-corrected visual acuity (BCVA); 2.corticosteroid- 
sparing effect; 3.Central Macular Thickness (CMT); 4.Adverse events

Data management: Two authors will independently extract data from the included publications. Any discrepancies will be resolved through discussion and referral to a third reviewer if needed. A standardised piloted data extraction form will be used. Study authors may be contacted if further information is required. For each study, the following information (but not limited to) will be extracted.

Quality assessment / Risk of bias analysis: Quality assessment of all included articles will be under-taken by two reviewers independently with disagreements resolved by discussion and referral to a third reviewer if required. We will independently assess the risk of bias as described in the Cochrane Handbook for Systematic Reviews of Interventions. The following items will be assessed: random sequence generation; allocation concealment; blinding; incomplete outcome data; selective outcome reporting; other bias. In case of any unresolved disagreements, MSL will make the final decision as arbiter.

Strategy of data synthesis: All statistical analyses will be performed using Review Manager software (version 5.3). Continuous data will be reported as mean difference with $95 \%$ Cls. For primary outcomes, if the meta-analysis results are significantly heterogeneous, subgroup analysis will be performed as detailed below. $\circ$ We will use the Grading of Recommendations Assessment, Development and Evaluation (GRADE) software to determine the quality of evidence based on the Cochrane Handbook for Systematic Reviews oflnterventions to create a Summary of Findings table. We will use the random effect model for meta-analysis. If a metaanalysis is possible, we will use the $I^{2}$ statistic to quantify inconsistencies across the included studies. A resulting $50 \%$ cut-off point would represent substantial heterogeneity. Where heterogeneity is exited, we will conduct subgroup analyses.
Subgroup analysis: Where studies and data permit, subgroup analyses will be conducted according to the types of uveitis, duration of treatment or patients' ages.

Sensibility analysis: In the case of sufficient trials data, the risk of bias tool will be used to assess methodological quality. If lowquality articles are deleted, a second metaanalysis will be performed. The results and effect size of the two meta-analyses will be compared and discussed.

Language: No language restriction.

Country(ies) involved: China.

Keywords: noninfectious uveitis; adalimumab; efficacy; safety; randomized controlled trials.

Contributions of each author:

Author 1 - Biao Li - Conceptualization; Data curation; Formal analysis; Methodology; Writing - original draft; Writing - review \& editing.

Author 2 - Haoran Li - Data curation; Methodology; Writing - original draft.

Author 3 - Li Zhang - Formal analysis; Methodology; Writing - original draft.

Author 4 - Yanlin Zheng - Project administration; Supervision; Writing review \& editing. 